\title{
Gellan Nanohydrogels: Novel Nanodelivery Systems for Cutaneous Administration of Piroxicam
}

\author{
3 Umberto M. Musazzi, ${ }^{\dagger}$ Claudia Cencetti, ${ }^{\ddagger}$ Silvia Franzé, ${ }^{\dagger}$ Nicole Zoratto, ${ }^{\ddagger}$ Chiara Di Meo, ${ }^{\ddagger}$ \\ ${ }_{4}$ Patrizia Procacci, ${ }^{\S}$ Pietro Matricardi, ${ }^{\ddagger}{ }^{\ddagger}$ and Francesco Cilurzo $*^{\dagger}+(1)$ \\ $5{ }^{\dagger}$ Department of Pharmaceutical Sciences, University of Milan, via Giuseppe Colombo, 71, 20133 Milan, Italy \\ 6 "Department of Drug Chemistry and Technologies, "Sapienza” University of Rome, Piazzale Aldo Moro 5, 00185, Rome, Italy \\ $7{ }^{\S}$ Department of Biomedical Sciences for Health, University of Milan, via Giuseppe Colombo, 71, 20133 Milan, Italy
}
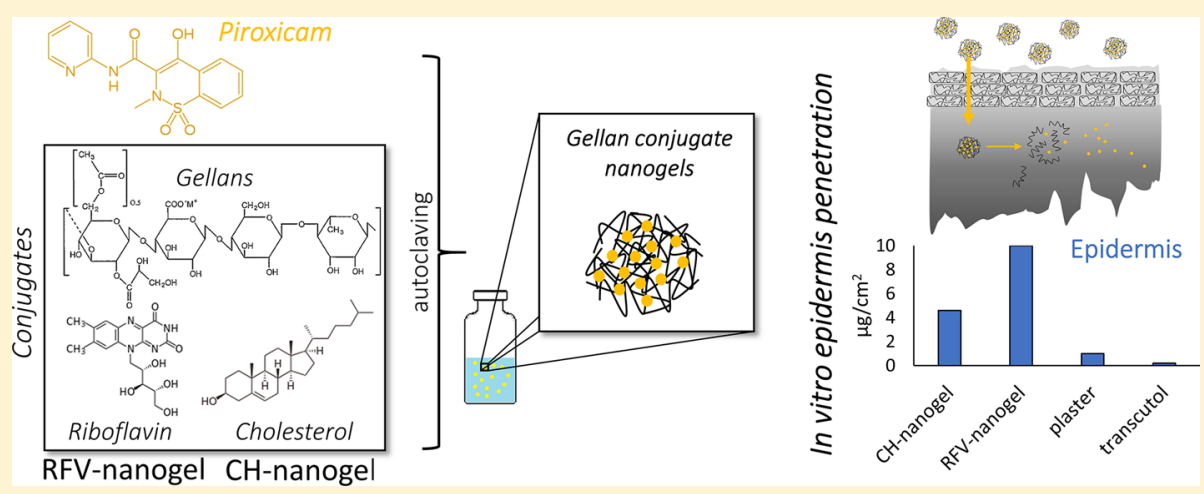

9 ABSTRACT: The feasibility to use gellan nanohydrogels (Ge-NH) as delivery system for the cutaneous administration of 10 piroxicam (PRX) was investigated using gellan conjugated with cholesterol or riboflavin. The in vitro skin penetration studies 11 through human epidermis were performed using a saturated aqueous drug solution, a 50\% w/v Transcutol aqueous solution, and 12 a commercially available PRX plaster as controls. Confocal microscopy, ATR-FTIR spectroscopy, circular dichroism, and a 13 dynamometer assisted extrusion assay were performed to clarify the permeation mechanism of Ge-NHs. The skin permeation 4 studies evidenced that Ge-NHs enhance the PRX retention in the epidermis and, at the same time, slow down the permeation process with respect to the controls. NHs can penetrate the stratum corneum, and then gradually disassemble thus diffusing in the viable epidermis reaching the spinosum layer. In conclusion, NHs represent a novel strategy to target poorly permeable compounds in the epidermis, thus improving the management of cutaneous pathologies.

8 KEYWORDS: nanohydrogel, gellan, self-assembling polysaccharide, piroxicam, skin penetration

\section{$19 \square$ INTRODUCTION}

20 Nanosize hydrogels (nanogels, NHs) are polymer nanoparticles 21 with three-dimensional networks, formed by chemical and/or 22 physical cross-linking of polymer chains. Various nanogels have 23 been designed as drug-delivery systems (DDS) and studied for 24 different biomedical applications, such as regenerative medicine 25 and bioimaging applications. ${ }^{1-4}$

26 Very recently, the feasibility to administer a drug in the skin 27 by the use of nanogels has been also proposed. ${ }^{5-8}$ Indeed, 28 having a highly porous structure with a large water content, $29 \mathrm{NHs}$ seem to be able to enhance the skin penetration of the 30 embedded (mainly hydrophobic) compounds. As an example, 31 Smejkalova and co-workers found a significant increase of the 32 retention of a hydrophobic probe (i.e., nile red) both in 33 epidermis and in dermis using a nanogels obtained from an 34 esterified hyaluronic acid. ${ }^{9}$ In another work, the nano35 encapsulation of ibuprofen into a ternary complex with chitosan 36 and gellan significantly increased the permeation of the drug with respect to the control hydrogel. ${ }^{10}$ Analogously, the 37 permeation of acitretin through pig ear skin was improved by 38 chitin base nanogels. 5 The skin permeation profile can be 39 further optimized by using stimulus responsive nanogels as 40 demonstrated in the case of caffeine. ${ }^{11}$

The permeation mechanism of nanogel through the skin has 42 not been yet completely clarified, and, basically, two different 43 hypotheses are reported in the literature. According to the first 44 one, the enhancement effect may be related to the softness and 45 physicochemical stability of the nanogel as suggested in the case 46 of polyglycerol-based nanogels. ${ }^{12}$ Alternatively, a loss of 47 nanogel integrity with skin depth and a copenetration of 48 nanogel components and their payload has been suggested. ${ }^{9} \quad 49$

Received: October 24, 2017

Revised: December 21, 2017

Accepted: January 24, 2018

Published: January 25, 2018 
50 In order to further support the efficacy of physically cross51 linked nanogels in enhancing the skin penetration of drugs, 52 providing more information on the mechanism aspects, this 53 work aimed to explore the feasibility of gellan based 54 nanohydrogels (Ge-NHs) to deliver piroxicam (PRX) in 55 human epidermis.

56 The feasibility to obtain Ge-NHs by using an innovative, 57 reproducible process based on a standard autoclave cycle and 58 the physicochemical stability of the resulting systems have been 59 already demonstrated. ${ }^{13-16} \mathrm{NH}$ formation results from the self60 assembling of $\mathrm{Ge}$-cholesterol $(\mathrm{GeCh})$ and Ge-riboflavin 61 (GeRfv) derivatives, and hydrophobic drugs can be easily 62 loaded within Ge-NHs. ${ }^{14}$ PRX appears of particular interest as 63 model drug since it is a very poorly permeable compound if 64 compared to the other nonsteroidal antinflammatory drugs ${ }^{17}$ 65 and it has been proposed for the treatment of nonmelanoma 66 skin cancers. ${ }^{18,19}$

67 Due to the risk of serious gastrointestinal adverse effects in 68 comparison to other NSAIDs, ${ }^{20,21}$ the development of a 69 therapeutic system able to enhance the PRX retention in the 70 skin, reducing at the same time the systemic exposure, may be 71 beneficial to improve the safety of the treatment.

72 In the present work, the in vitro permeation profiles through 73 human epidermis of PRX-loaded GeCh-NHs and GeRfv-NHs 74 were investigated. Aiming to better understand their possible 75 application in the design of drug delivery systems intended for 76 cutaneous applications, their penetration patterns were 77 compared to those obtained with a PRX saturated aqueous 78 solution, PRX Transcutol aqueous solution, and a commercially 79 available medicated plaster. The solutions were selected as 80 reference to determine how the penetration profile of PRX was 81 influenced by the drug loading in the nanohydrogel. In 82 particular, Transcutol was used since it is a well-known skin 83 penetration enhancer which favors the retention in the skin. ${ }^{22}$ 84 The medicated plaster was selected to better understand how 85 efficient were Ge-NHs in enhancing drug permeation with 86 respect to a drug product already authorized for the market for 87 the treatment of locoregional painful syndromes.

\section{EXPERIMENTAL SECTION}

89 Materials. Piroxicam (PRX) was purchased from Cameo 90 Healthcare (Thane, India). Egg phosphatidylcholine (Egg-PC) 91 was kindly provided by Lipoid (Steinhausen, Switzerland). 92 Tween 80 was obtained from Croda chocques (France); 93 HPLC-grade, acetonitrile, and $\mathrm{MeOH}$ were purchased from 94 VWR International PBI s.r.l. (Milan, Italy). Gellan (Ge) (Kelko 95 product) was purchased from Giusto Faravelli (Italy). 96 Cholesterol $(\mathrm{Ch})$ and riboflavin (Rfv) were Sigma-Aldrich Srl 97 (Milan, Italy) products. Transcutol (diethylene glycol mono98 ethyl ether) was obtained from Gattefossé Italia (Milan Italy). 99 All other reagents and solvents were purchased from Sigma100 Aldrich Srl (Milan, Italy) and used without further purification. 101 Synthesis of Cholesterol- and Riboflavin-Conjugated 102 Gellans. The high molecular weight native Ge (sodium salt 103 form) was, at first, modified in the tetrabutylammonium salt 104 form by ion-exchange resin. To obtain NHs, it is necessary to 105 lower the $M_{\mathrm{W}}$ of the polymer. To this aim, Ge was extruded 106 through an orifice at high pressure conditions (M-110EH 107 Microfluidizer Processor in an extrusion chamber G10Z, 87 $108 \mathrm{~mm} ; P 1200$ bar, $T=50{ }^{\circ} \mathrm{C}$ ). A final $M_{\mathrm{W}} \approx 2.75 \times 10^{5}$ 109 determined by GPC was obtained. ${ }^{13}$

110 As a general method, the Ge derivatives were obtained by 111 esterifying the polymer chains with a hydrophobic moiety, previously derivatized using an appropriate spacer according to 112 a previously described reaction. Gellan-cholesterol ( $\mathrm{GeCh}$ ) was 113 obtained by linking cholesterol (esterified with 4-bromobutyric 114 acid; $\mathrm{Ch}$ ) to the polymer chains in $N$-methyl pyrrolidone 115 (derivatization degree, $9 \% \mathrm{~mol} / \mathrm{mol}$ ). ${ }^{13}$ Gellan-riboflavin 116 (GeRfv) was obtained by linking riboflavin tetrabutyrate 117 (functionalized with 1,6-dibromohexane, $\mathrm{Rfv}$ ) to the polymer 118 chains (derivatization degree, by feed ratio, $30 \% \mathrm{~mol} / \mathrm{mol}$ ) 119 according to the reaction already described in the literature. ${ }^{15} 120$ Polymer derivatives were characterized according to procedures 121 already described. ${ }^{23}$ Moreover, FTIR spectra $\left(4000-650 \mathrm{~cm}^{-1}\right){ }_{122}$ showed for both the samples a peak at about $1750 \mathrm{~cm}^{-1}, 123$ indicating the formation of the ester linkage between $\mathrm{Ge}$ and 124 the hydrophobic moieties (Figure S1).

125

Preparation of Nanogels. The NHs were prepared by the 126 autoclave process as previously reported. ${ }^{14,15}$ Briefly, $3.0 \mathrm{mg}$ of 127 each gellan derivative (i.e., $\mathrm{GeCh}$ or $\mathrm{GeRfv}$ ) were dispersed in 128 $3.0 \mathrm{~mL}$ of distilled water $(1.0 \mathrm{mg} / \mathrm{mL})$ by overnight magnetic 129 stirring at $25{ }^{\circ} \mathrm{C}$. At the same time, $1 \mathrm{~mL}$ of PRX acetone 130 solution $(2 \mathrm{mg} / \mathrm{mL})$ was added to a glass vial and stored at 131 room temperature until the complete solvent evaporation, thus 132 forming a film of the drug. The Ge derivate suspension $(3 \mathrm{~mL}){ }_{133}$ was then added to this vial, shaken in vortex, and autoclaved for 134 $20 \mathrm{~min}$ at $121^{\circ} \mathrm{C}$ and 1.10 bar (Autoclave-steam sterilizer 2440135 ML, Tuttnauer, NL). At the end of the process, a PRX-loaded 136 $\mathrm{NH}$ suspension was obtained. The resulting suspension was 137 withdrawn and centrifuged at $20{ }^{\circ} \mathrm{C}, 4000 \mathrm{rpm}, 10 \mathrm{~min} 138$ (Universal 30RF, Hettich Zentrifugen, Germany). The super- 139 natant containing NHs was stored for further studies. Blank 140 NHs were prepared as well following the same protocol. The 141 PRX chemical stability to the autoclaving process was 142 preliminary evaluated by the HPLC method reported below. 143

Particle Size and $\zeta$-Potential Measurements. The mean 144 hydrodynamic diameter of NHs $\left(D_{\mathrm{h}}\right), \mathrm{PDI}$, and $\zeta$-potential 145 were measured at $25{ }^{\circ} \mathrm{C}$ by the DLS method. Aliquots of 146 supernatant containing NHs were diluted 1:10 in HPLC-grade 147 water and analyzed by Zetasizer Nano ZS (Malvern Instru- 148 ments Ltd., U.K.).

Drug Content. The PRX amount loaded in the NHs was 150 determined indirectly as follows. After the centrifugation step 151 above-described, the pellet was solubilized in $2 \mathrm{~mL}$ of ethanol 152 to extract the unloaded amount of PRX. At the same time, the 153 vial used for autoclave cycle was cleaned with $2 \mathrm{~mL}$ of ethanol 154 to recover the PRX absorbed on the wall. The two ethanolic 155 solutions were mixed and diluted 1:20 in the mobile phase 156 described below to determine the PRX amount unloaded. The 157 amount of the loaded drug was determined by subtracting the 158 result from the amount of PRX initially added to the vial. 159

Absorption Spectrum of PRX. The determination of 160 maximum peak of absorbance $\left(\lambda_{\max }\right)$ of PRX loaded in the NHs 161 was performed using a UV-vis spectrometer Lambda 25162 (PerkinElmer, USA). Before the analyses, the drug-loaded NH 163 suspension was diluted 1:10 in HPLC-grade water and, then, 164 put in quartz cuvettes with a light path of $10 \mathrm{~mm}$ (Hellma 165 Analytics, USA). The UV spectrum was recorded from 200 to 166 $700 \mathrm{~nm}$ using HPLC-grade water as blank reference. The $\lambda_{\max } 167$ was graphically determined from the resulting UV spectrum of 168 the sample. Saturated PRX aqueous solution and PRX solutions 169 $(20 \mu \mathrm{g} / \mathrm{mL})$ in acidified $(0.125 \mathrm{M}$ phosphate buffer sodium $\mathrm{pH} 170$ 2.0, Eur.Ph. 9.0; $\mathrm{pH}=2)$ and alkaline conditions ( $0.1 \mathrm{M}_{171}$ phosphate buffer solution, Eur.Ph. 9.0; $\mathrm{pH}=10$ ) were used as 172 controls. 
174 Mechanical Properties of Nanogels. The ability of NHs 175 to act as soft carriers, squeezing through the narrow 176 intracellular spaces of the packed stratum corneum, was assessed 177 by a dynamometer assisted extrusion process, according to the 178 method previously developed for studying the deformability of 179 flexible liposomes. ${ }^{24}$ Briefly, samples of Ge-NHs were diluted to 180 a concentration of $0.05 \mathrm{mg} / \mathrm{mL}$ in Milli-Q water and loaded in $1811 \mathrm{~mL}$ gastight syringe, held with the needle facing downward 182 into an extruder casing fixed to a vertical holder. The syringe 183 plunger was put in touch with a $50 \mathrm{~N}$ load cell of a 184 dynamometer (INSTRON 5965, ITW Test and Measurement 185 Italia Srl, Italy) set up to move the plunger at a constant rate of $1861 \mathrm{~mm} / \mathrm{s}$, forcing $\mathrm{NH}$ suspension through a polycarbonate 187 membrane, pore diameter $50 \mathrm{~nm}$. To minimize the dead 188 volume and to carry out the test correctly, the membrane was 189 prewetted with Milli-Q water before each test. At the end of the 190 experiment the extruded fraction was collected and analyzed by 191 DLS for particle size determination and counting of the 192 particles. The force $(\mathrm{N})$ required to extrude the $\mathrm{NH}$ suspension 193 through the membrane was registered as a function of the 194 plunger displacement ( $\mathrm{mm})$, and the constant of deformability 195 ( $k$ ) was calculated as the slope of the resulting curve. ${ }^{24}$ A highly 196 flexible transferosome formulation, made of Egg-PC and Tween $19780(85: 15 \mathrm{w} / \mathrm{w} \%)$ and prepared by the thin film hydration 198 method, was used as a reference.

199 Quantification of Piroxicam by HPLC. The concen200 tration of PRX was determined by HPLC (HP 1100, 201 ChemStations, Agilent Technologies, USA). Separation was 202 carried out using a reverse-phase column (LiChrospher 100 $203 \mathrm{RP}-18 \mathrm{E}, 5 \mu \mathrm{m}, 125 \times 4.0 \mathrm{~mm}$, CPS Analitica, Italy) and $0.03 \mathrm{M}$ 204 phosphate $\mathrm{pH} 3.0$ buffer/acetonitrile $(60 / 40 \% \mathrm{v} / \mathrm{v})$ as mobile 205 phase. The flow rate was $1.5 \mathrm{~mL} / \mathrm{min}$, and the injection volume 206 was $20 \mu \mathrm{L}$. The retention time of PRX was $2.4 \mathrm{~min}$. The drug 207 concentration was determined at two wavelengths (i.e., $248 \mathrm{~nm}$, $208360 \mathrm{~nm}$ ) from calibration curves in the range of $0.05-20 \mu \mathrm{g} /$ $209 \mathrm{~mL}\left(R^{2}>0.999\right)$.

210 In Vitro Skin Permeation Experiments. The permeation 211 studies were performed using the frozen abdominal skin from 212 three different donors, who underwent cosmetic surgery within 213 one month before the in vitro experiment. Epidermis samples 214 were prepared following an internal standard procedure. ${ }^{25}$ The 215 full-thickness skin was sealed in evacuated plastic bags and 216 frozen at $-20{ }^{\circ} \mathrm{C}$ within $6 \mathrm{~h}$ after removal. Before the 217 experiments, the skin was thawed at room temperature, and the 218 excess of fat was carefully removed. The skin sections were cut 219 into squares of about $4.9 \mathrm{~cm}^{2}$, and, after immersion in water at $22060{ }^{\circ} \mathrm{C}$ for $1 \mathrm{~min}$, the epidermis was gently separated from the 221 remaining tissue with forceps. The electrical resistance $(R)$ of 222 the isolated epidermis was measured to ensure the integrity of 223 the barrier membrane, and epidermis samples with $R$ values 224 above $25 \mathrm{k} \Omega \mathrm{cm}^{2}$ were used for experiments (voltage, $100 \mathrm{mV}$; 225 frequency, $100 \mathrm{~Hz}$; Agilent 4263B LCR Meter, Microlease, I). 226 The epidermis sample was mounted on the Franz diffusion cell 227 with a permeation area of $0.636 \mathrm{~cm}^{2}$. The receptor compart228 ment (volume: $\approx 3.0 \mathrm{~mL}$ ) was filled with $0.9 \% \mathrm{w} / \mathrm{v} \mathrm{NaCl}$ 229 aqueous solution containing $100 \mu \mathrm{g} / \mathrm{mL} \mathrm{NaN}_{3}$ as preservative. 230 The receptor phase was selected to guarantee the sink 231 condition throughout the permeation experiments. Special 232 care was given to avoid air bubbles between the buffer and the 233 epidermis in the receptor compartment. The upper and lower 234 parts of the Franz cell were sealed with Parafilm (Pechiney 235 Plastic Packaging Company, USA) and fastened together by 236 means of a clamp, with the epidermis acting as a seal between the donor and receptor compartments. Then, the donor 237 compartment was filled with $0.4 \mathrm{~mL}$ of tested liquid 238 formulations. The system was kept at $37{ }^{\circ} \mathrm{C}$ using a circulating 239 water bath, so that the epidermis surface temperature was at 32240 $\pm 1{ }^{\circ} \mathrm{C}$ throughout the experiment. At predetermined time 241 intervals $(1,4,6,16,24 \mathrm{~h}), 200 \mu \mathrm{L}$ samples were withdrawn 242 from the receiver compartment and replaced with a fresh 243 aliquot of receiver medium. Samples were analyzed by HPLC 244 according to the method described in the dedicated section. 245 Moreover, in the case of GeRfv-NHs, the withdrawn aliquots 246 were also analyzed using a Wallac 1420 Victor2Microplate 247 Reader (PerkinElmer, US) to estimate the permeation pattern 248 of the GeRfv. The excitation and the emission wavelengths 249 were set at 355 and $535 \mathrm{~nm}$, respectively. The values are 250 expressed as the average of parallel experiments performed at 251 least in duplicate using an epidermis sheet from each of the 252 three donors $(n=6)$. The cumulative amount permeated 253 through the skin per unit area $\left(Q_{i}\right)$ was calculated from the 254 drug concentration in the receiving medium and plotted as a 255 function of time. The maximal flux $\left(J_{\max }\right)$ was determined as the 256 slope of the linear portion of the plot $Q_{i}$ vs time $\left(R^{2}>0.98\right) .257$

At the end of the permeation experiments, the concentration 258 of PRX retained in the epidermis $\left(R_{24}\right)$ was quantified by the 259 following procedure. The epidermis sheet was removed from 260 the Franz diffusion cell, and each side was gently treated with 5261 $\mathrm{mL}$ of methanol $(\mathrm{MeOH})$ to wash out the unabsorbed drug. 262 Subsequently, the sample was dried, thinly sliced, and placed in 263 $5 \mathrm{~mL}$ of fresh $\mathrm{MeOH}$. The suspension was soaked in a 264 sonicator for $30 \mathrm{~min}$ and then maintained for $24 \mathrm{~h}$ at $2-8{ }^{\circ} \mathrm{C} .265$ Finally, the supernatant was filtered through a $0.45 \mu \mathrm{m}$ filter 266 and analyzed by HPLC. $R_{24}$ was expressed as micrograms of 267 PRX per square centimeter.

The $R_{24} / Q_{24}$ ratio was used to underline the PRX affinity for 269 the epidermal layers and the influence of the formulation on 270 such paramenter. ${ }^{26}$

The performances of the NHs in terms of skin permeation 272 were assessed using a PRX saturated aqueous $(16 \mu \mathrm{g} / \mathrm{mL}) 273$ solution, $500 \mu \mathrm{g} / \mathrm{mL}$ PRX in a Transcutol aqueous solution 274 $(50 \% \mathrm{w} / \mathrm{v})$, and a medicated plaster already available on the 275 Italian market (PRX content $200 \mu \mathrm{g} / \mathrm{cm}^{2}$; Brexidol, Promedica 276 Srl, Italy). In this case, the experimental protocol was slightly 277 modified: despite liquid formulation, the plaster was attached 278 on the human epidermis immediately before the membrane was 279 mounted on the Franz diffusion cell.

Attenuated Total Reflection (ATR) Spectroscopy. 281 Epidermis samples were prepared following an internal 282 standard procedure as described above. $24 \mathrm{~h}$ before the ATR 283 analyses, each epidermis sample was put in a Petri dish and 2.0284 $\mathrm{mL}$ of $1 \mathrm{mg} / \mathrm{mL} \mathrm{NH}$ aqueous dispersion was added at the 285 interface with the stratum corneum. An aliquot of Milli-Q grade 286 water was used as control. The samples were stored at room 287 temperature for $24 \mathrm{~h}$. At the end of incubation time, each 288 sample was carefully cleaned to eliminate the solution residues 289 on the surface and, then, dried at room temperature for $30 \mathrm{~min} 290$ before ATR analyses. Afterward, each sample was carefully 291 wiped with filter paper and let equilibrate for $15 \mathrm{~min}$ at $23{ }^{\circ} \mathrm{C} 292$ and 50\% RH before testing. FTIR measurements were 293 performed using a SpectrumOne spectrophotometer (Perki- 294 nElmer, USA), by placing the epidermal sample on a diamond 295 crystal mounted in an ATR cell (PerkinElmer, USA). The 296 spectra were collected over the wavenumber region 4000-650 297 $\mathrm{cm}^{-1}$ at $4 \mathrm{~cm}^{-1}$ resolution and 128 scans. The raw data were 298 elaborated by ATR correction (Spectrum, PerkinElmer, USA); 299 
300 the band maxima were assigned by second derivative after 301 smoothing with a seven-point Savitsky-Golay function using 302 Originlab 2015.

303 Circular Dichroism (CD). To detect possible interaction 304 between the Ge derivatives and keratins in the stratum corneum, $305 \mathrm{CD}$ spectra were recorded using regenerated keratin as model 306 compound. The protein was obtained by wool using the 307 protocol described by Selmin and co-workers. ${ }^{27} \mathrm{CD}$ spectra 308 were recorded on a J-810 spectropolarimeter, using a quartz 309 cuvette of $0.1 \mathrm{~cm}$ path length, between 190 and $260 \mathrm{~nm}$ at a 310 scanning rate of $50 \mathrm{~nm} / \mathrm{min}$ with $0.5 \mathrm{~nm}$ resolution. The 311 analyses were carried out on $0.1 \mathrm{mg} / \mathrm{mL}$ samples in water at 312 room temperature.

313 Confocal Microscopy Analysis. Samples from in vitro 314 permeation experiments were embedded in OCT compound 315 (Miles Laboratories, Elkhart, IN, USA), rapidly frozen in liquid 316 nitrogen, and stored at $-80{ }^{\circ} \mathrm{C}$, pending sectioning. Cryostat 317 sections ( $15 \mu \mathrm{m}$ thick), cut from frozen skin samples, were 318 collected on adhesive coated slides, air-dried, and stored at -20 $319{ }^{\circ} \mathrm{C}$ until use.

320 For confocal microscopy analysis, sections were rehydrated 321 in PBS $0.1 \mathrm{M}$ and stained with 4',6-diamidino-2-phenylindole 322 (DAPI), Sigma-Aldrich Srl (Milan, Italy), to label nuclei. After 323 final washing, sections were mounted with Mowiol mounting 324 medium Sigma-Aldrich Srl (Milan, Italy).

325 Sections were examined with a confocal microscope Zeiss 326 LSM 510 system (Gottingen, Germany).

327 Images from different slices were obtained through a $40 \times$ oil 328 immersion objective at $1024 \times 1024$ pixel resolution, and 329 acquired with the software A.I.M. 4.2.

\section{$330 \square$ RESULTS}

331 Characterization of Nanogels. The two prepared NHs, 332 based on GeCh and GeRfv, had similar physicochemical 333 characteristics. Indeed, $D_{\mathrm{h}}$ were $177 \pm 28$ and $178 \pm 16 \mathrm{~nm}$ for $334 \mathrm{GeCh}-\mathrm{NH}$ s and GeRfv-NHs, respectively. The $\zeta$-potential was 335 close to $-40 \mathrm{mV}$ in both cases, determining a good physical 336 stability in water. The PRX was uploaded within the NHs by 337 autoclaving a suspension of the drug in the presence of the $\mathrm{Ge}$ 338 derivatives. The PRX content resulted as $282 \pm 31 \mu \mathrm{g} / \mathrm{mg}$ and $339301 \pm 12 \mu \mathrm{g} / \mathrm{mg}$, respectively. It is worth noticing that the 340 more general mechanism of the uploading of hydrophobic 341 drugs within the NHs is not yet clearly elucidated. As described 342 in a previous paper, ${ }^{16}$ the mechanism could be related to the 343 hydrophobic interactions among the drug molecules and the 344 hydrophobic moieties responsible of the $\mathrm{NH}$ assemblies. These 345 interactions could be favored by the high temperature reached 346 during the autoclave treatment. It should be also emphasized 347 that the preparation method led to obtaining $\mathrm{NH}$ suspensions 348 in which the drug was mainly loaded into the nanogel, but also 349 partially free in the suspension medium, which resulted as 350 saturated, avoiding the drug leakage from the nanocarrier. In 351 addition, the results obtained by UV analyses highlighted that 352 the encapsulation of PRX in NHs did not alter its ionization 353 equilibrium since the $\lambda_{\max }$ of both saturated solution and PRX354 loaded GeCh-NHs resulted as superimposable (Table 1). The 355 tests were performed only on GeCh-NHs, as the $\lambda_{\max }$ of GeRfv 356 interfered with the absorbance spectrum of PRX.

357 Moreover, the obtained $\lambda_{\max }$ values were comparable to that 358 obtained at $\mathrm{pH} 10$, suggesting that PRX was in the zwitterionic/ 359 anionic form. Indeed, it is noteworthy that the $\lambda_{\max }$ of PRX 360 varies as a function of the ionization state of the molecule. ${ }^{28}$ At 361 acidic $\mathrm{pH}$ values, the pyridine moiety, that is part of the PRX
Table 1. Maximum Peak of Absorbance of PRX in Nanogels and Reference Solutions

\begin{tabular}{llc}
\multicolumn{1}{c}{ formulation } & \multicolumn{1}{c}{ solvent } & $\lambda_{\max }(\mathrm{nm})$ \\
satd PRX soln & water & 358 \\
PRX-loaded GeCh-NHs & water & 357 \\
PRX soln pH 2 & acidified water $(\mathrm{pH}=2)$ & 343 \\
PRX soln pH 10 & alkaline water $(\mathrm{pH}=10)$ & 354 \\
\hline
\end{tabular}

structure, results to be protonated. When the $\mathrm{pH}$ increases 362 above 7 , the deprotonation of pyridine moiety induces a 363 bathochromic shift of $\lambda_{\max }$. Although the PRX anionic form 364 prevails at high alkaline $\mathrm{pH}$, the stabilization of a planar 365 conformation of PRX due to the intramolecular hydrogen bond 366 between the pyridine and the carbonyl group permits the 367 formation of zwitterion. With respect to the experimental data, 368 the existence of PRX zwitterionic form was also suggested by 369 the yellowish color observed in the case of the saturated PRX 370 solution. $^{29}$

In Vitro Skin Permeation Experiments. The permeation 372 profile of saturated PRX solution resulted to be negligible 373 (Table 2; Figures S2, S3), despite the $R_{24}$ value suggesting that 374 t2 PRX penetrates the stratum corneum. Indeed, it is noteworthy 375 that the low drug solubility in water strongly influences the 376 permeation process, affecting the concentration gradient among 377 the membrane layers. On the contrary, the PRX content in the 378 medicated plaster induced a 25-fold increase of the amount of 379 the drug loaded in the donor compartment with respect to the 380 saturated solution. In these conditions, the PRX significantly 381 permeated through the human epidermis, as demonstrated by 382 the resulting flux value and the retained amount (Table 2; 383 Figures S2, S3). However, $Q_{24}$ and $R_{24}$ were $1.04 \pm 0.28 \%$ and 384 $0.52 \pm 0.14 \%$ of the applied dose respectively, confirming the 385 limited poor tendency of PRX to permeate through the skin. 386

When NHs were used as drug carriers, the $R_{24}$ values 387 obtained after the application of NHs significantly increased 388 with respect to the reference formulations (Table 2, p-value 389 $<0.05)$. Moreover, the permeation process was slowed down in 390 comparison to medicated plaster $\left(Q_{24}, J_{\max }\right.$, lag time, $p$-values 391 $<0.001$ ). Independently of the composition of the NHs, a slight 392 increase of the "PRX utilization" of the dosage form with 393 respect to the medicated plaster was recorded. In agreement 394 with the EMA Guideline on quality of transdermal patch 395 (EMA/CHMP/QWP/608924/2014), the PRX utilization was 396 defined as the percentage of total drug absorbed within the 397 entire in vitro experiment with respect to the initial drug loading 398 of the dosage form. Indeed, the PRX utilization of the 399 medicated plaster was $\approx 1.5 \%$ while that of the NHs resulted at 400 least 2-fold higher. In particular, GeRfv-NHs appeared to be the 401 most efficient formulation with respect to the $R_{24}$ expressed 402 both as absolute value (Table 2) and as percentage (i.e., $5.59 \pm 403$ $2.03 \%$ of the applied dose). In addition, the dose-normalized 404 permeation results showed that GeRfv-NHs was effective in 405 minimizing the drug permeation through the human epidermis 406 (i.e., $0.03 \pm 0.01 \%$ ). The capability of $\mathrm{NHs}$ to promote the 407 PRX retention within the human epidermis was also confirmed 408 by the comparison of the $R_{24} / Q_{24}$ values (Table 2). If the 409 plaster value was bias for the permeation through the skin $\left(R_{24} / 410\right.$ $\left.Q_{24}=0.5\right)$, the $R_{24} / Q_{24}$ was strongly in favor of the numerator 411 of the fraction for all the $\mathrm{NH}$ formulations $\left(R_{24} / Q_{24}>20\right)$. This 412 resulted in a GeRfv-NHs ratio 10 times higher than $\mathrm{GeCh}-413$ NHs. 
Table 2. Results of in Vitro Permeation Studies on PRX-Loaded GeCh-NHs, GeRfv-NHs, PRX 50\% w/v Transcutol Solution, and Saturated Solution and Plaster (Mean Value \pm SD; $n=6$ )

\begin{tabular}{lcccccc}
\multicolumn{1}{c}{ formulation } & PRX dose $\left(\mu \mathrm{g} / \mathrm{cm}^{2}\right)$ & $\mathrm{Q}_{24}\left(\mu \mathrm{g} / \mathrm{cm}^{2}\right)$ & lag time $(\mathrm{h})$ & flux $\left(\mu \mathrm{g} / \mathrm{cm}^{2} / \mathrm{h}\right)$ & $R_{24}\left(\mu \mathrm{g} / \mathrm{cm}^{2}\right)$ & $R_{24} / Q_{24}$ \\
GeCh-NHs & 146 & $0.221 \pm 0.107$ & $7 \pm 4$ & $0.018 \pm 0.005$ & $4.626 \pm 1.237$ \\
GeRfv-NHs & 189 & $0.049 \pm 0.020$ & $>16$ & $-{ }^{a}$ & $10.290 \pm 3.743$ & 210 \\
satd soln & 8 & - & $>24$ & - & $0.031 \pm 0.053$ \\
50\% w/v Transcutol soln & 200 & $0.436 \pm 0.167$ & $>16$ & $-{ }^{a}$ & - \\
plaster & 200 & $2.07 \pm 0.551$ & $3 \pm 0$ & $0.100 \pm 0.024$ & $1.033 \pm 0.275$
\end{tabular}

${ }^{a_{\text {The }}}$ flux was not calculated since the long lag time.

415 Based on the obtained results, the GeRfv-NHs, which 416 resulted as the most effective formulation in enhancing the 417 localization of PRX in the skin, was thus selected to study the 418 penetration pattern.

419 Confocal Microscopy Images. The pattern of penetration 420 of the GeRfv-NHs within the human epidermis was followed by 421 confocal microscopy, taken advantage of the autofluorescence 422 of riboflavin. Images were recorded on skin samples exposed to 423 the GeRfv-NHs for different times (1, 2, 4, 6, 24 h; Figure 1). 424 Nuclei of the epidermal cells appear as bright blue ovals. In 425 control samples (not shown) any green signal was evident.

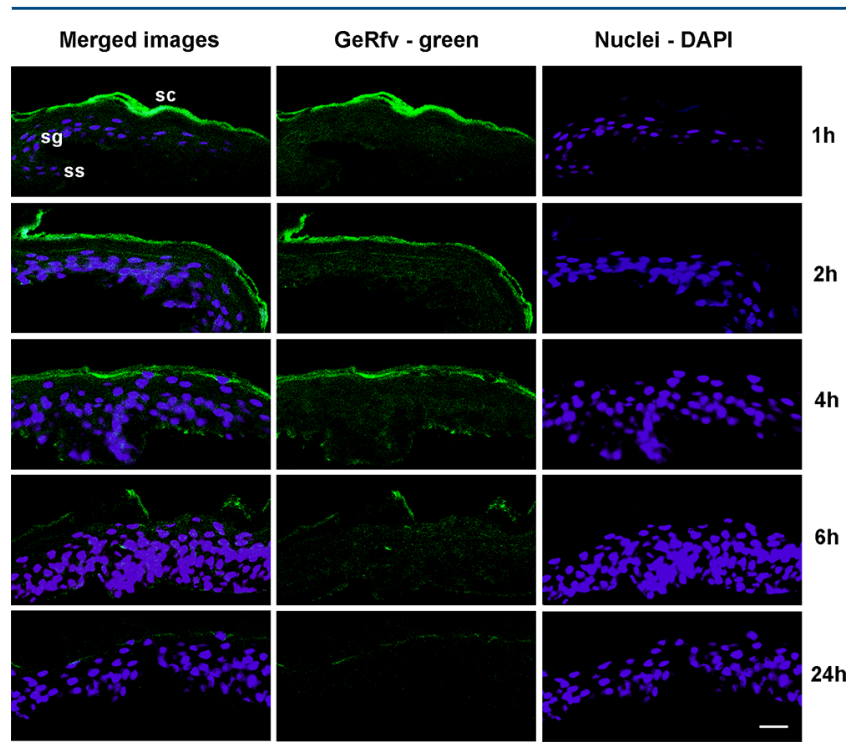

Figure 1. Confocal images obtained from frozen skin samples treated by GeRfv-NHs. Merged images show green signal detection on the outer surface of the skin and in the deeper layers at different times points after the treatment. Green fluorescence is due to the GeRfv. Nuclei are stained blue with DAPI. SC = stratum corneum; SG = stratum granulosum; SS = stratum spinosum. Images were acquired under a $40 \times$ oil immersion objective. Scale bar $=20 \mu \mathrm{m}$.

426 As depicted in Figure 1, after $1 \mathrm{~h}$ of exposure, NHs 427 concentrate mainly on the outer skin surface, remaining 428 essentially confined in the stratum corneum, while a weak 429 fluorescence is noticed in the epidermal layers, mainly at the 430 granulosum-spinosum limit (after $2 \mathrm{~h}$ ). The probe signal, 431 detected on the outer surface of the skin, underwent a 432 significant reduction over time until it almost disappeared after $43324 \mathrm{~h}$ after the initial treatment. This seems to be related to the 434 diffusion of the probe deeper in the epidermis layers, up to the 435 spinosum layer. Indeed, after $6 \mathrm{~h}$ exposure to GeRfv-NHs a 436 homogeneous green fluorescent signal was observed through437 out the epidermis, suggesting a great affinity of the 438 polysaccharide scaffolding of the gel for the skin components.
Finally, it should be mentioned that the fluorescence of GeRfv- 439 $\mathrm{NHs}$ in the receiving medium of the Franz cell after $24 \mathrm{~h} 440$ experiment was not significantly different from the fluorescence 441 of the samples exposed to untreated epidermis samples, 442 suggesting a negligible GeRfv permeation through the human 443 epidermis.

Mechanical Properties of Nanogels. Nanosized channel- 445 like pores of the stratum corneum are one of the major 446 pathways suggested for the skin penetration of nanocarriers, ${ }^{9} 447$ and, therefore, the ability of the delivery systems to squeeze 448 through these pores is a requirement for the release of the drug 449 payload deep into the skin. Nanogels are highly porous, soft, 450 and compressible materials, since they can exchange water with 451 the external environment undergoing swelling and deswelling 452 processes. Therefore, they could adapt their structure thus 453 crossing the tight skin barrier. In this respect, Montanari et al. 454 found that HA based nanogels exhibit a low Young's modulus 455 as determined by AFM analysis. ${ }^{16}$ Moreover, Hendrickson and 456 Lyon reported the first proof of concept that nanogels, after 457 application of a pressure, can undergo such a deformation to 458 pass through cylindrical pores having a diameter one tenth 459 smaller than their own diameter. ${ }^{30}$ Basing on this evidence, we 460 investigated the behavior of our NHs during constriction 461 passage through track-etch membranes having pores at least 3.5462 times smaller than their own size to resemble the penetration 463 through the nanoporous structure of the stratum corneum. The 464 test was performed at constant rate to estimate the forces acting 465 during this process. In Figure 2, a representative curve obtained $466 \mathrm{f} 2$ after extrusion of the NHs is reported.

After the first peak, which corresponds to the force required 468 to overcome the frictions for plunger displacement, the force 469

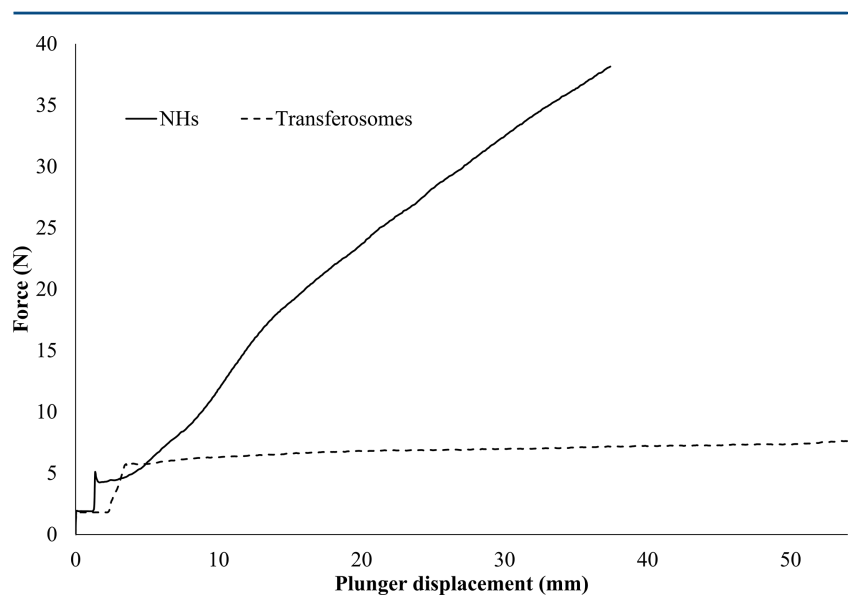

Figure 2. Representative curves of the force versus plunger displacement registered forcing GeRfv-NHs through $50 \mathrm{~nm}$ polycarbonate membranes (solid line) and transferosomes (dashed line). 
Table 3. ATR-FTIR Data of Human Epidermis Samples Treated with GeCh-NHs and GeRfv-NHs ${ }^{a}$

\begin{tabular}{|c|c|c|c|c|c|c|c|}
\hline \multirow[b]{2}{*}{ epidermis treatment } & \multicolumn{2}{|c|}{ stretching $\mathrm{CH}_{3}$} & \multicolumn{2}{|c|}{ stretching $\mathrm{CH}_{2}$} & \multirow[b]{2}{*}{$H_{2920 / 2850}$} & \multirow[b]{2}{*}{ scissoring $\mathrm{CH}_{x}$} & \multirow[b]{2}{*}{ fwhm } \\
\hline & symmetric & asymmetric & symmetric & asymmetric & & & \\
\hline control & $2958.0 \pm 0.1$ & $2923.5 \pm 0.1$ & $2873.3 \pm 0.1$ & $2854.1 \pm 0.1$ & $1.841 .0 \pm 0.1$ & $1466.4 \pm 0.1$ & $12.1 \pm 0.3$ \\
\hline GeCh-NHs & $2958.1 \pm 0.1$ & $2923.2 \pm 0.1$ & $2873.4 \pm 0.1$ & $2854.0 \pm 0.1$ & $1.783 .0 \pm 0.0$ & $1466.5 \pm 0.0$ & $12.8 \pm 0.8$ \\
\hline GeRfv-NHs & $2957.8 \pm 0.3$ & $2923.1 \pm 0.1$ & $2873.2 \pm 0.1$ & $2854.1 \pm 0.1$ & $1.801 .0 \pm 0.0$ & $1466.6 \pm 0.1$ & $13.6 \pm 0.3$ \\
\hline
\end{tabular}

${ }^{a}$ Epidermis samples treated only by water were used as a control.

470 progressively increased until the maximum limit (based on the 471 equipment performances) was reached determining the end of 472 the test run. Only $2 \%$ of NHs were found in the filtrate and the 473 particle size resulted reduced by about $40 \%$ of the initial particle 474 diameter, suggesting that nanogels partially lost their structure 475 during the passage through the pores, finally occluding the 476 porous membrane. Indeed, the force versus plunger displace477 ment curve registered for NHs did not present the plateau 478 segment registered for transferosome suspension, which seems 479 to freely flow through the membrane pores (Figure 2). Finally, 480 the $k$ value registered for the $\mathrm{NHs}(\sim 0.9 \mathrm{~N} / \mathrm{mm})$ resulted to be 481 4-fold higher than that obtained for Egg-PC based trans482 ferosomes $(\sim 0.02 \mathrm{~N} / \mathrm{mm})$.

483 ATR-FTIR Spectroscopy and CD Measurements. The 484 changes in the barrier properties of stratum corneum exposed to 485 a chemical can be attributed to an alteration of the intercellular 486 lipids from orthorhombic conformation toward the less ordered 487 hexagonal conformation and/or the extraction of ceramides. 488 These effects can be monitored by ATR-FTIR spectroscopy 489 analyzing the possible shift of bands in the $\mathrm{CH}_{x}$ stretching 490 region or measuring the $\mathrm{CH}_{2}$ scissoring bandwidth (expressed 491 as the full width at $50 \%$ peak height, fwhm). ${ }^{31}$ The treatment of 492 the human epidermis by nanogels did not determine any 493 significant shift of $\mathrm{CH}_{x}$ stretching vibration bands (Table 3; 494 Figure S3). Moreover, the fwhm values of the $\mathrm{CH}_{2}$ scissoring 495 bandwidth were in the $11.0-14.0 \mathrm{~cm}^{-1}$ range, confirming that 496 all formulations did not affect the organization of lipids since 497 they remained in the most stable orthorhombic conformation. 498 This lack of alteration of lipidic network was further confirmed 499 by the evaluation of the ratio between the heights of the band at 500 about 2923 and $2853\left(H_{2920 / 2850}\right)$ which, remaining almost 501 constant after the treatment with $\mathrm{NHs}$, excluded a possible 502 extraction of lipids from the stratum corneum.

503 The analysis of the amide I and amide II regions confirmed 504 the lack of significant interactions between the Ge-NHs and the 505 main stratum corneum components. First, the treatment of 506 epidermis sheet with Ge-NHs does not determine any 507 modification of the maximum of the amide I that remained 508 centered in the $1650-1660 \mathrm{~cm}^{-1}$ region, confirming that the 509 main conformation of $\alpha$-helical conformations prevails in the 510 stratum corneum proteins (data not shown). Second, the ratio 511 among the amide I and amide II height, which is an indication 512 of protein unfolding, ${ }^{32}$ remained almost constant. Third, the 513 research of the amide I hidden peaks by second derivative 514 evidenced the lack of any significant modification of the 515 wavelengths, which overlapped those already described in the 516 literature. ${ }^{33}$ This wavenumber did not significantly change after 517 the treatment by both of the formulations. This lack of 518 significant modifications in keratin conformations is further 519 confirmed by $\mathrm{CD}$ analyses performed on regenerated wool 520 keratin which presents a prevalent $\alpha$-helical conformation. 521 Indeed, the CD spectra recorded on regenerated keratin and 522 keratin $\mathrm{GeCh}$ mixtures overlapped and the small reduction of the intensity was attributed to dilution of the protein, as $523 \mathrm{f} 3$ exemplified in Figure 3.

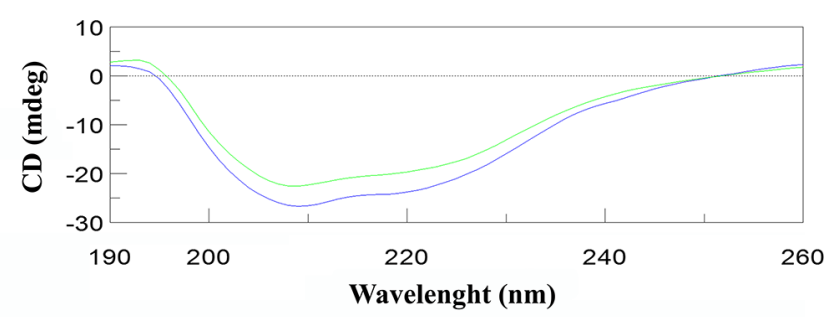

Figure 3. $\mathrm{CD}$ spectra of regenerated keratin (blue line) and regenerated keratin/GeCh $1 / 1$ mixture (green line).

\section{DISCUSSION}

Ge-NHs represent a new useful tool in drug delivery for many 526 reasons. The biocompatibility of Ge and the soft consistency of 527 the NHs, due to the hydrogel structure at nanoscale, is a 528 favorable environment for loading drugs in a well-tolerated 529 device. Moreover, the innovative method for the NHs forming 530 and loading in one shot by means of an autoclave, leading to a 531 sterile suspension, is particularly appealing for a large-scale 532 application of these nanodevices. Ge-NHs have been already 533 exploited in loading and delivery of hydrophobic drugs, such as 534 paclitaxel. In that case, the drug loading is allowed by the 535 hydrophobic interactions between the drug molecules and the 536 hydrophobic zones in the NHs, which are built up by the 537 cholesterol moieties linked to the polymer chains. As can be 538 easily argued, this property can be exploited for loading and 539 delivery of many other hydrophobic drugs. Starting from the 540 above-mentioned properties and taking into account the skin 541 structure and the results described in the literature on the 542 applications of nanodevices in topical drug delivery, ${ }^{5,7,8}$ this 543 work aims to demonstrate the usefulness of Ge-NHs also in 544 cutaneous applications, improving the PRX administration 545 through the skin. The PRX loading in Ge-NHs determined a 546 modification of its skin penetration pattern with respect to a 547 medicated plaster, which can be considered one of the most 548 effective dosage forms to sustain the permeation of this model 549 drug through the skin. Indeed, NHs enhanced the skin 550 retention, $R_{24} / Q_{24}$ values being significantly higher than those 551 obtained by both the Transcutol solution and the PRX plaster. 552 The same effect was also described in the case of clobetasol: the 553 use of chitin nanogels that enhanced the skin retention with 554 respect to a marketed cream. ${ }^{7}$ The comparison with Transcutol 555 is particularly interesting since this compound is claimed to 556 increase the drug solubility in the skin and therefore its 557 retention.

This data suggested that the effect of nanogels cannot merely 559 be ascribed to an increase of the PRX apparent solubility. 560 Indeed, a modification of the drug apparent solubility should 561 influence only the partition of the permeant from the donor 562 
563 toward the stratum corneum while the subsequent processes 564 (i.e., the diffusion through the epidermis layers and the 565 partition toward the receiver medium) should resulted 566 unaffected. Thus, assuming that the adhesive matrix of a 567 patch acts as a drug reservoir on the skin, ${ }^{34}$ the $R_{24} / Q_{24}$ values 568 obtained with the Ge-NHs and the medicated plaster should 569 overlap. Moreover, the enhancement of the localization of PRX 570 in the epidermis layer could not be ascribed to a change in the 571 ionic equilibrium of PRX, as shown by the negligible differences 572 between the UV spectrum of native PRX and drug-loaded $573 \mathrm{GeCh}-\mathrm{NH}$. Such findings seem to demonstrate that Ge-NHs 574 play an active role in the whole permeation process of PRX 575 through the human epidermis. As clearly evidenced by confocal 576 images, the green signal of Rfv was already detectable in the 577 stratum granulosum after $1 \mathrm{~h}$ from the application. At time 578 points of 2,4 , and $6 \mathrm{~h}$ the signal became detectable also in the 579 stratum spinosum, even if weaker and more scattered. However, 580 since the resolution confocal microscope cannot discriminate 581 the presence of intact NHs into the epidermis, the generated 582 data are unable to define if the Ge derivatives are able or not to 583 penetrate the skin as intact $\mathrm{NHs}$ or disassembled during the 584 penetration. Although these results are in agreement with other 585 published ones, ${ }^{6,7}$ no conclusive evidence was available 586 regarding the penetration mechanism of nanogels.

587 It may be hypothesized that, once Ge-NHs are in contact 588 with the skin environment, a modification of their inner 589 structure may occur. Indeed, hydrophobic attractive forces 590 between the hydrophobic moieties of $\mathrm{Ge}$ chains (i.e., 591 cholesterol, riboflavin) stabilized the 3D structure of Ge-NHs 592 from a critical value of concentration in water. ${ }^{16}$ When such 593 systems interact with the upper skin layers, the low water 594 content $(\approx 20 \%)$ of stratum corneum and the presence of the 595 crystalline lipid matrix in the extracellular spaces ${ }^{35}$ may induce a 596 change of the interaction pattern within the spherical-like 597 network of Ge-NHs. In particular, the lipidic matrix of the 598 stratum corneum could interact with the hydrophobic moieties 599 of $\mathrm{Ge}$ derivatives (i.e., cholesterol, riboflavin), reducing the 600 cohesive forces between the polysaccharide chains and thus 601 favoring their disassembly.

602 The low water content of stratum corneum could also drive a 603 loss of water and a partial shrinkage of the swollen Ge-NHs. 604 Moreover, most of the green signal associated with the $\mathrm{Ge}$ 605 grafted riboflavin is localized in the stratum corneum and 606 appeared uniformly distributed suggesting an affinity for keratin 607 as already demonstrated for hyaluronan, which has a similar 608 structure. ${ }^{36}$ Thus, it is reasonable to hypothesize that the $\mathrm{NH}$ 609 shrinkage and the possible interactions with keratins could 610 trigger the disaggregation of the nanoassembly. This possibility 611 is supported by the measurements of the $\zeta$-potentials of 612 regenerated keratin, Ge derivative, and their mixtures. As an 613 example, the $\zeta$-potential of $0.1 \mathrm{mg} / \mathrm{mL}$ regenerated keratin and $614 \mathrm{GeCh}$ solutions was $-28.0 \pm 1.1 \mathrm{mV}$ and $-39.6 \pm 1.4 \mathrm{mV}$, 615 respectively. Their 1:1 mixture resulted as $-31.9 \pm 2.4 \mathrm{mV}$, 616 suggesting a possible weak interaction between the two 617 components, which reduced the $\zeta$-potential value of the 618 polysaccharide derivative. This interaction should mainly 619 influence the conformation of the Ge derivative since the CD 620 spectra did not evidence any modification of the regenerated 621 keratin and the ATR-FTIR spectra registered on the human 622 epidermis did not evidence any significant modification of the 623 conformation of skin proteins. This latter data appeared of 624 interest since the unfolding of skin protein is generally considered an indication of the possible toxicity of the 625 permeants. $^{32}$

All of the above considerations supported the idea that the 627 NHs favor the partition of PRX within the stratum corneum and 628 the $\mathrm{NH}$ disassembly can favor the localization of the drug in the 629 viable epidermis. The disassembly of the nanostructure could 630 also be justified by the reduction of the intensity of the green 631 signal in the confocal images of the epidermis layer over time. 632 This hypothesis is in agreement with the results of FRET 633 analyses performed on hydrophobic hyaluronic nanogels which 634 suggested their significant disaggregation when they reach the 635 viable epidermis. 9 Indeed, the nanogel did not present a 636 sufficient deformability, as demonstrated by the extrusion assay, 637 to penetrate the stratum corneum through the hydrophilic 638 pathway similarly to deformable vesicle and their dimensions 639 are too large for a diffusion through an intercellular route as in 640 the case of metallic nanoparticles. ${ }^{37}$ On the other hand, their 641 Young modulus is quite similar to that reported for 642 conventional liposomes, thus it was reasonable to hypothesize 643 that, even if the structure is completely different, their behavior 644 could be similar. Indeed, also in the case of conventional 645 liposomes it is generally recognized that they enhance the drug 646 retention into the skin disintegrating or fusing on the skin 647 surface, thus exchanging lipid components with the stratum 648 corneum and favoring the drug release. ${ }^{38}$

649

\section{CONCLUSIONS}

The literature has described the NHs as good drug carriers in 651 many applications. The results obtained in the present work 652 indicate that Ge-NHs can be useful in carrying and enhancing 653 the skin penetration of insoluble and poorly permeable drug 654 substances. Although their mechanism of action needs a deeper 655 investigation, the obtained data suggest that they can penetrate 656 intact in the upper skin layers and they experience a gradual 657 disassembling process by the diffusion in the deeper epidermis 658 layers. The ability of $\mathrm{GeCh}$ - and GeRfv-NHs to localize the 659 drug in the epidermis along with the possibility to be 660 internalized in epidermal cell lines makes such nanosystems 661 advantageous in opening new therapeutic strategies in the 662 management of cutaneous pathologies. Finally, it should be 663 underlined that these formulations could be considered as the 664 final dosage form to dermal delivery of drug molecules. Indeed, 665 in a previous work, we demonstrate the feasibility to administer 666 other nanocarriers, namely, ultraflexible liposomes and nano- 667 emulsions, by a simple spray. ${ }^{31,39}$ This approach should be 668 advantageous since the nanogel suspension can be administered 669 as such, avoiding dilutions that can reduce their concentration 670 or can cause the diffusion of the loaded drug outside the 671 nanocarrier. Indeed, the preparation method led to obtain a 672 suspension in which the drug is both loaded in the nanogel and 673 free in the suspension medium, which resulted as saturated, 674 avoiding drug leakage from the nanocarrier. In the specific case 675 of PRX, the permeation of the solubilized fraction through the 676 human epidermis is negligible in comparison to the penetration 677 pattern of PRX-loaded Ge-NHs, as demonstrated by the in vitro 678 skin permeation experiment performed using the saturated 679 solution.

\section{ASSOCIATED CONTENT}

The Supporting Information is available free of charge on the 683 ACS Publications website at DOI: 10.1021/acs.molpharma- 684 ceut.7b00926. 
686 FTIR spectra, PRX permeation profiles and retention

687 data, and ATR-FTIR spectra (PDF)

\section{AUTHOR INFORMATION}

\section{Corresponding Author}

690 *Phone: +39 02503 24635. Fax: +39 02503 24657. E-mail:

691 francesco.cilurzo@unimi.it.

692 ORCID

693 Pietro Matricardi: 0000-0003-2086-911X

694 Francesco Cilurzo: 0000-0003-3560-291X

695 Notes

696 The authors declare no competing financial interest.

\section{ACKNOWLEDGMENTS}

698 The authors would thank Dr. Simona Melfi for her assistance 699 during the acquisition of the confocal images. This research was 700 partially funded by Sapienza University, "Finanziamenti di 701 Ateneo per la Ricerca Scientifica-Anno 2016" and Progetto di 702 Ricerca RM11715C1743EE89.

\section{ABBREVIATIONS USED}

704 AFM, atomic force microscope; ATR, attenuated total 705 reflection; CD, circular dichroism; DAPI, 4',6-diamidino-2706 phenylindole; Egg-PC, egg phosphatidylcholine; Ge, gellan; $707 \mathrm{GeCh}$, gellan-cholesterol; GeRfv, gellan-riboflavin; $J_{\max }$ max708 imal flux; MetOH, methanol; NHs, nanohydrogels; NSAIDs, 709 nonsteroidal anti-inflammatory drugs; PDI, polydispersity 710 index; PRX, piroxicam; $Q_{i}$, cumulative amount permeated 711 through the skin per unit area after a period; $R$, electrical 712 resistance; $R_{24}$, drug retained amount into the epidermis after $71324 \mathrm{~h} ; \lambda_{\max }$ maximum peak of absorbance

\section{$714 \square$ REFERENCES}

715 (1) Chacko, R. T.; Ventura, J.; Zhuang, J.; Thayumanavan, S. 716 Polymer nanogels: A versatile nanoscopic drug delivery platform. Adv. 717 Drug Delivery Rev. 2012, 64, 836-851.

718 (2) Jiang, Y.; Chen, J.; Deng, C.; Suuronen, E. J.; Zhong, Z. Click 719 hydrogels, microgels and nanogels: Emerging platforms for drug 720 delivery and tissue engineering. Biomaterials 2014, 35, 4969-4985.

721 (3) Maya, S.; Sarmento, B.; Nair, A.; Rejinold, N. S.; Nair, S. V.; 722 Jayakumar, R. Smart stimuli sensitive nanogels in cancer drug delivery 723 and imaging: A review. Curr. Pharm. Des. 2013, 19, 7203-7218.

724 (4) Zhang, H.; Zhai, Y.; Wang, J.; Zhai, G. New progress and 725 prospects: The application of nanogel in drug delivery. Mater. Sci. Eng., 726 C 2016, 60, 560-568.

727 (5) Divya, G.; Panonnummal, R.; Gupta, S.; Jayakumar, R.; Sabitha, $728 \mathrm{M}$. Acitretin and aloe-emodin loaded chitin nanogel for the treatment 729 of psoriasis. Eur. J. Pharm. Biopharm. 2016, 107, 97-109.

730 (6) Kim, J.; Gauvin, R.; Yoon, H. J.; Kim, J. H.; Kwon, S. M.; Park, H. 731 J.; Baek, S. H.; Cha, J. M.; Bae, H. Skin penetration-inducing gelatin 732 methacryloyl nanogels for transdermal macromolecule delivery. 733 Macromol. Res. 2016, 24 (12), 1115-1125.

734 (7) Panonnummal, R.; Jayakumar, R; Sabitha, M. Comparative anti735 psoriatic efficacy studies of clobetasol loaded chitin nanogel and 736 marketed cream. Eur. J. Pharm. Sci. 2017, 96, 193-206.

737 (8) Zabihi, F.; Wieczorek, S.; Dimde, M.; Hedtrich, S.; Borner, H. G.; 738 Haag, R. Intradermal drug delivery by nanogel-peptide conjugates; 739 specific and efficient transportation of temoporfin. J. Controlled Release 740 2016, 242, 35-41.

741 (9) Šmejkalová, D.; Muthný, T.; Nešporová, K.; Hermannová, M.; 742 Achbergerová, E.; Huerta-Angeles, G.; Svoboda, M.; Čepa, M.; 743 Machalová, V.; Luptáková, D.; Velebny, V. Hyaluronan polymeric 744 micelles for topical drug delivery. Carbohydr. Polym. 2017, 156, 86-96.
(10) Abioye, A. O.; Issah, S.; Kola-Mustapha, A. Ex vivo skin 745 permeation and retention studies on chitosan-ibuprofen-gellan 746 ternary nanogel prepared by in situ ionic gelation technique-a tool 747 for controlled transdermal delivery of ibuprofen. Int. J. Pharm. 2015, 748 490, 112-130.

749

(11) Samah, N. H. A.; Heard, C. M. Enhanced in vitro transdermal 750 delivery of caffeine using a temperature- and $\mathrm{pH}$-sensitive nanogel, 751 poly(NIPAM-co-AAc). Int. J. Pharm. 2013, 453, 630-640.

752

(12) Rancan, F.; Asadian-Birjand, M.; Dogan, S.; Graf, C.; Cuellar, L.; 753 Lommatzsch, S.; Blume-Peytavi, U.; Calderón, M.; Vogt, A. Effects of 754 thermoresponsivity and softness on skin penetration and cellular 755 uptake of polyglycerol-based nanogels. J. Controlled Release 2016, 228, 756 159-169.

(13) D’Arrigo, G.; Navarro, G.; Di Meo, C.; Matricardi, P.; Torchilin, 758 V. Gellan gum nanohydrogel containing anti-inflammatory and anti- 759 cancer drugs: a multi-drug delivery system for a combination therapy 760 in cancer treatment. Eur. J. Pharm. Biopharm. 2014, 87 (1), 208-216. 761 (14) Montanari, E.; De Rugeriis, M. C.; Di Meo, C.; Censi, R.; 762 Coviello, T.; Alhaique, F.; Matricardi, P. One-step formation and 763 sterilization of gellan and hyaluronan nanohydrogels using autoclave. J. 764 Mater. Sci.: Mater. Med. 2015, 26, 32.

765

(15) Di Meo, C.; Montanari, E.; Manzi, L.; Villani, C.; Coviello, T.; 766 Matricardi, P. Highly versatile nanohydrogel platform based on 767 riboflavin-polysaccharide derivatives useful in the development of 768 intrinsically fluorescent and cytocompatible drug carriers. Carbohydr. 769 Polym. 2015, 115, 502-509.

(16) Montanari, E.; Di Meo, C.; Sennato, S.; Francioso, A.; Marinelli, 771 A. L.; Ranzo, F.; Schippa, S.; Coviello, T.; Bordi, F.; Matricardi, P. 772 Hyaluronan-cholesterol nanohydrogels: Characterisation and effective- 773 ness in carrying alginate lyase. New Biotechnol. 2017, 37, 80-89. 774 (17) Cilurzo, F.; Gennari, C. G. M.; Selmin, F.; Franzè, S.; Musazzi, 775 U. M.; Minghetti, P. On the characterization of medicated plasters 776 containing NSAIDs according to novel indications of USP and EMA: 777 adhesive property and in vitro skin permeation studies. Drug Dev. Ind. 778 Pharm. 2015, 41, 183-189.

(18) Campione, E.; Diluvio, L.; Paternò, E. J.; Chimenti, S. Topical 780 Treatment of Actinic Keratoses with Piroxicam 1\% Gel. Am. J. Clin 781 Derm 2010, 11, 45-50.

(19) Campione, E.; Paterno, E. J.; Candi, E.; Falconi, M.; Costanza, 783 G.; Diluvio, L.; Terrinoni, A.; Bianchi, L.; Orlandi, A. The relevance of 784 piroxicam for the prevention and treatment of nonmelanoma skin 785 cancer and its precursors. Drug Des., Dev. Ther. 2015, 9, 5843-5850. 786 (20) Henry, D.; McGettigan, P. Epidemiology overview of gastro- 787 intestinal and renal toxicity of NSAIDs. Int. J. Clin. Pract., Suppl. 2003, 788 $135,43-49$.

(21) Laporte, J. R.; Ibanez, L.; Vidal, X.; Vendrell, L.; Leone, R. 790 Upper gastrointestinal bleeding associated with the use of NSAIDs: 791 newer versus older agents. Drug Saf. 2004, 27, 411-420. 792

(22) Godwin, D. A.; Kim, N. H.; Felton, L. A. Influence of 793 Transcutol ${ }^{\circledR}$ CG on the skin accumulation and transdermal 794 permeation of ultraviolet absorbers. Eur. J. Pharm. Biopharm. 2002, 795 53 (1), 23-27.

(23) D’Arrigo, G.; Di Meo, C.; Gaucci, E.; Chichiarelli, S.; Coviello, 797 T.; Capitani, D.; Alhaique, F.; Matricardi, P. Self-assembled gellan- 798 based nanohydrogels as a tool for prednisolone delivery. Soft Matter 799 2012, 8, 11557-11564.

(24) Franzé, S.; Donadoni, G.; Podestà, A.; Procacci, P.; Orioli, M.; 801 Carini, M.; Minghetti, P.; Cilurzo, F. Tuning the extent and depth of 802 penetration of flexible liposomes in human skin. Mol. Pharmaceutics 803 2017, 14 (6), 1998-2009.

804

(25) Musazzi, U. M.; Matera, C.; Dallanoce, C.; Vacondio, F.; De 805 Amici, M.; Vistoli, G.; Cilurzo, F.; Minghetti, P. On the selection of an 806 opioid for local skin analgesia: Structure-skin permeability relation- 807 ships. Int. J. Pharm. 2015, 489, 177-185.

808

(26) Franzé, S.; Gennari, C. G. M.; Minghetti, P.; Cilurzo, F. 809 Influence of chemical and structural features of low molecular weight 810 heparins (LMWHs) on skin penetration. Int. J. Pharm. 2015, 481 (1- 811 2), 79-83. 
813 (27) Selmin, F.; Cilurzo, F.; Aluigi, A.; Franzè, S.; Minghetti, P. 814 Regenerated keratin membrane to match the in vitro drug diffusion 815 through human epidermis. Results Pharma Sci. 2012, 2 (1), 72-78.

816 (28) Rozou, S.; Voulgari, A.; Antoniadou-Vyza, E. The effect of $\mathrm{pH}$ 817 dependent molecular conformation and dimerization phenomena of 818 piroxicam on the drug: cyclodextrin complex stoichiometry and its 819 chromatographic behaviour. A new specific HPLC method for 820 piroxicam:cyclodextrin formulations. Eur. J. Pharm. Sci. 2004, 21, $821661-669$.

822 (29) Tsai, R.-S.; Carrupt, P.-A.; Tayar, N. E.; Giroud, Y.; Andrade, P.; 823 Testa, B.; Brée, F.; Tillement, J.-P. Physicochemical and structural 824 properties of non-steroidal anti-inflammatory oxicams. Helv. Chim. 825 Acta 1993, 76, 842-854.

826 (30) Hendrickson, G. R.; Lyon, L. A. Microgel translocation through 827 pores under confinement. Angew. Chem., Int. Ed. 2010, 49, 2193-2197. 828 (31) Campani, V.; Biondi, M.; Mayol, L.; Cilurzo, F.; Franzé, S.; 829 Pitaro, M.; De Rosa, G. Nanocarriers to Enhance the Accumulation of 830 Vitamin K1 into the Skin. Pharm. Res. 2016, 33 (4), 893-908.

831 (32) Karande, P.; Jain, A.; Ergun, K.; Kispersky, V.; Mitragotri, S. 832 Design principles of chemical penetration enhancers for transdermal 833 drug delivery. Proc. Natl. Acad. Sci. U. S. A. 2005, 102 (13), 4688-93. 834 (33) Gennari, C. G. M.; Franzè, S.; Pellegrino, S.; Corsini, E.; Vistoli, 835 G.; Montanari, L.; Minghetti, P.; Cilurzo, F. Skin Penetrating Peptide 836 as a Tool to Enhance the Permeation of Heparin through Human 837 Epidermis. Biomacromolecules 2016, 17 (1), 46-55.

838 (34) Cilurzo, F.; Selmin, F.; Gennari, C. G. M.; Montanari, L.; 839 Minghetti, P. Application of methyl methacrylate copolymers to the 840 development of transdermal or loco-regional drug delivery systems. 841 Expert Opin. Drug Delivery 2014, 11 (7), 1033-1045.

842 (35) Damien, F.; Boncheva, M. The extent of orthorhombic lipid 843 phases in the stratum corneum determines the barrier efficiency of 844 human skin in vivo. J. Invest. Dermatol. 2010, 130, 611-614.

845 (36) Cilurzo, F.; Vistoli, G.; Gennari, C.; Selmin, F.; Gardoni, F.; 846 Franzè, S.; Campisi, M.; Minghetti, P. The role of the conformational 847 profile of polysaccharides on skin penetration: the case of hyaluronan 848 and sulfates thereof. Chem. Biodiversity 2014, 11 (3), 551-561.

849 (37) Musazzi, U. M.; Santini, B.; Selmin, F.; Marini, V.; Corsi, F.; 850 Allevi, R.; Ferretti, A. M.; Prosperi, D.; Cilurzo, F.; Colombo, M.; 851 Minghetti, P. Impact of semi-solid formulations on skin penetration of 852 iron oxide nanoparticles. J. Nanobiotechnol. 2017, 15, 14-20.

853 (38) Cosco, D.; Celia, C.; Cilurzo, F.; Trapasso, E.; Paolino, D. 854 Colloidal carriers for the enhanced delivery through the skin. Expert 855 Opin. Drug Delivery 2008, 5 (5), 737-755.

856 (39) Campani, V.; Biondi, M.; Mayol, L.; Cilurzo, F.; Pitaro, M.; De 857 Rosa, G. Development of nanoemulsions for topical delivery of 858 vitamin K1. Int. J. Pharm. 2016, 511, 170-177. 